\title{
Near-field error sensing for active directivity control of radiated sound
}

Shuping Wang, Hongmei Sun, Jie Pan, and Xiaojun Qiu

Citation: The Journal of the Acoustical Society of America 144, 598 (2018); doi: 10.1121/1.5049145

View online: https://doi.org/10.1121/1.5049145

View Table of Contents: http://asa.scitation.org/toc/jas/144/2

Published by the Acoustical Society of America

\section{Articles you may be interested in}

Subwavelength and quasi-perfect underwater sound absorber for multiple and broad frequency bands

The Journal of the Acoustical Society of America 144, 648 (2018); 10.1121/1.5048797

An active control strategy for the scattered sound field control of a rigid sphere

The Journal of the Acoustical Society of America 144, EL52 (2018); 10.1121/1.5046446

REVIEWS OF ACOUSTICAL PATENTS

The Journal of the Acoustical Society of America 144, 536 (2018); 10.1121/1.5048415

Acoustic radiation force on an elastic sphere in a soft elastic medium

The Journal of the Acoustical Society of America 144, 568 (2018); 10.1121/1.5047442

Mechanisms of active control of sound radiation from an opening with boundary installed secondary sources

The Journal of the Acoustical Society of America 143, 3345 (2018); 10.1121/1.5040139

Low-frequency band gap of locally resonant phononic crystals with a dual-base plate

The Journal of the Acoustical Society of America 143, 1326 (2018); 10.1121/1.5025041 


\title{
Near-field error sensing for active directivity control of radiated sound
}

\author{
Shuping Wang, ${ }^{1, a)}$ Hongmei Sun, ${ }^{2}$ Jie Pan, ${ }^{2}$ and Xiaojun Qiu $^{3}$ \\ ${ }^{1}$ Key Laboratory of Modern Acoustics and Institute of Acoustics, Nanjing University, Nanjing 210093, \\ People's Republic of China \\ ${ }^{2}$ School of Mechanical and Chemical Engineering, The University of Western Australia, Perth, \\ Western Australia 6009, Australia \\ ${ }^{3}$ Centre for Audio, Acoustics and Vibration, Faculty of Engineering and IT, University of Technology Sydney, \\ Ultimo, New South Wales 2007, Australia
}

(Received 3 May 2018; revised 4 July 2018; accepted 17 July 2018; published online 6 August 2018)

\begin{abstract}
Near-field error sensing is beneficial to the compactness and stability of an active noise control system. This paper proposes an error sensing strategy based on the spatial Fourier transform to achieve active directivity control of radiated sound. The error microphone array is located on a plane close to the primary source and the cost function is the weighted sum of the error signals from the microphones. The weighting factor is related to the phase shift from the error microphones to the plane perpendicular to the direction where noise reduction is required. The geometric configurations of the error microphone array for effective directivity control are investigated. It is found that the distance between neighboring error microphones must be less than approximately half the wavelength of the frequency of interest and the equivalent size of the microphone array should be larger than twice the size of the primary source. Numerical simulations and experiments demonstrate the feasibility of the proposed strategy. (C) 2018 Acoustical Society of America.
\end{abstract} https://doi.org/10.1121/1.5049145

$[\mathrm{NK}]$

Pages: 598-607

\section{INTRODUCTION}

The physical configurations of an active noise control (ANC) system are very important as they determine the upper limit of the performance the system can achieve. To achieve global sound power reduction, secondary sources should be close to the primary source, for example, within half the wavelength from the primary source in the free field. ${ }^{1}$ Another important step in designing the physical part of an ANC system is to find the best positions for error microphones and a proper cost function for minimization.

To actively reduce the radiated sound power, microphones should provide an error signal that is proportional to the total radiated sound power. According to ISO 3744, the sound power level can be measured with 20 microphones on a hemisphere in a semi-anechoic chamber, and the radius of the test sphere should be equal to or greater than each of these three sizes: (1) twice the largest source dimension; (2) a quarter of the wavelength of interest; and (3) $1 \mathrm{~m}$. Therefore, it is not practical for use in ANC systems, especially at low frequencies or when the noise source is large. ${ }^{2}$ Another way to measure the total sound power is to use sound intensities measured in the near field of the noise sources, but Berry et al. found that the strategy of minimizing the near-field sound intensity suffers from two main limitations due to the fact that sound intensity is a signed quantity. ${ }^{3}$

In cases where control of total sound power is difficult or unnecessary, control of sound radiated into specified areas

${ }^{\text {a)} E l e c t r o n i c ~ m a i l: ~ w a n g s p 822105 @ 126 . c o m ~}$ offers an alternative. A commonly used error sensing strategy for local control is to minimize the sound pressure within a local area. ${ }^{4}$ Julliard et al. applied error sensors in the far field from an inlet fan and yielded up to $15 \mathrm{~dB}$ attenuation in a large angular range in the radial extension of the sensor location. ${ }^{5}$ Rafaely investigated the potential use of a spherical loudspeaker array for local active control of sound and created a larger quiet zone compared to a monopole source with the error microphones within the local area where noise reduction is required. ${ }^{6}$ All of the above error sensing strategies can create a local quiet zone; however, they cannot guarantee noise reduction in the far field.

Qiu and Zhao proposed to use the sum of the squared sound pressures at several points within an angle as the cost function and achieved directivity control from the near to the far field, but some of the error points are far from the primary source. ${ }^{7}$ For the compactness and stability of ANC systems, error microphones should be located in the near field of the primary and secondary sources. This paper will investigate a near-field error sensing strategy that can achieve directivity control in both the near and far fields. The motivation for investigating this method arises from an industry project, where the noise radiated from a hauling truck to a direction where a community locates is required to be reduced. ${ }^{8}$

The challenge of near-field error sensing is to find an appropriate cost function and the best positions for error microphones. For the active control of free field radiation, the optimal positions for error microphones are where the noise reduction is the greatest when the strengths of secondary sources are optimized by minimizing the total radiated 
sound power. ${ }^{9}$ Shafer et al. experimentally demonstrated that with the error sensors in theoretically ideal locations, the measured near-field sound pressure map approximates the theoretical map created when minimizing the radiated power, but moving them to non-ideal locations greatly reduces the attenuation of sound power. ${ }^{10}$

Previous work has reported much effort to find these ideal locations and the appropriate cost function. Zander and Hansen investigated several error sensing strategies, including the minimization of pressure at a point, the total real acoustic power output, an estimate of the acoustic potential energy, and a new error sensing strategy based on minimization of the power flow determined by a modal decomposition of the duct sound field and found that the best strategy is minimizing the downstream power flow. ${ }^{11}$ Qiu et al. compared eight different cost functions for nearfield error sensing of a monopole and dipole primary source and found that the most appropriate strategy is minimizing the sum of the weighted mean active intensity in a direction normal to the surface surrounding all the primary and control sources. ${ }^{12}$

Another near-field error sensing strategy is the virtual error sensor arrangement, where physical error microphones are located near the primary source to estimate the sound pressure at virtual error sensor locations far from it and create a quiet zone there, ${ }^{13}$ but it can only achieve local control. Berkhoff showed the effectiveness of applying virtual error sensors on an active noise barrier with numerical simulations. ${ }^{14}$ Fuller et al. applied the virtual error sensor technology on a portable generator set and achieved overall reductions of between 0.5 and $4.6 \mathrm{dBA}$ at virtual error sensor locations; however, because of the inaccuracy of the modeling of transfer functions, it is lower than the noise reduction at physical sensors (3.5-11 dBA). ${ }^{15}$ Peterson et al. investigated the virtual error sensing in a rigid-walled acoustic duct and found that theoretically it is possible to obtain infinite reductions at virtual locations and this can be extended to create a moving quiet zone, but the noise reduction will decrease for high excitation frequencies and larger virtual distances. ${ }^{16,17}$ Petersen et al. proposed a virtual sensing algorithm for local active noise control systems using Kalman filtering theory and demonstrated its effectiveness by implementing the algorithm on an acoustic duct arrangement. ${ }^{18} \mathrm{~A}$ problem with the virtual error sensor arrangement is that it requires preliminary identification of the system.

In this paper, a new near-field error sensing strategy is proposed for active directivity control of radiated sound. By implementing an array of near-field microphones in a plane close to the primary source, sound radiation in the desired direction is reduced in both the near and far fields. The requirements on the error microphone array are investigated with numerical simulations. The feasibility of the proposed method is demonstrated with experiments in an anechoic chamber. In practical applications, the error microphones can be located on a plane perpendicular to the direction where noise reduction is required, and by summing up the signals at all the microphones as one error signal, only a single channel active controller is needed, resulting in the low complexity and cost of the system.

\section{THEORY}

A schematic diagram of the near-field error microphone array is illustrated in Fig. 1. The array is located in front of the primary source on the $z=z_{0}$ plane with evenly distributed error microphones, and noise reduction is required in the direction defined by the unit vector $\mathbf{n}(\sin \theta \cos \varphi$, $\sin \theta \cos \varphi, \cos \theta$ ), where $\theta$ and $\varphi$ are shown in Fig. 1.

Based on the spatial Fourier transform, the amplitude of the sound in wavenumber domain can be expressed as an integral over the $z=z_{0}$ plane $^{19}$

$$
P\left(k_{x}, k_{y}, z_{0}\right)=\int_{-\infty}^{\infty} \int_{-\infty}^{\infty} p\left(x, y, z_{0}\right) \mathrm{e}^{j\left(k_{x} x+k_{y} y+k_{z} z_{0}\right)} \mathrm{d} x \mathrm{~d} y,
$$

where

$$
k_{x}^{2}+k_{y}^{2}+k_{z}^{2}=k^{2},
$$

$k=c / 2 \pi f$ is the wavenumber; $c$ is the sound speed in the air; $f$ is the frequency of interest; $p\left(x, y, z_{0}\right)$ is the sound pressure at any point $\left(x, y, z_{0}\right)$ in the $z=z_{0}$ plane; and $k_{x}, k_{y}$, and $k_{z}$ are the components of the wavenumber in $x, y$, and $z$ directions, respectively. Noise reduction in the direction of $\mathbf{n}$ is required, so $k_{x}, k_{y}$, and $k_{z}$ can be calculated with

$$
\begin{aligned}
& k_{x}=k \sin \theta \cos \varphi, \\
& k_{y}=k \sin \theta \sin \varphi, \\
& k_{z}=k \cos \theta,
\end{aligned}
$$

where $\theta \in(-\pi / 2, \pi / 2)$ and $\varphi \in[0,2 \pi)$. The amplitude of sound radiation in this direction can be calculated with Eqs. (1) and (3).

The sum of the weighted sound pressures at several sampling points in the $z=z_{0}$ plane is used to approximate the integral in Eq. (1). The squared amplitude of the sum of the sound pressures at $L$ error points evenly distributed on the $z=z_{0}$ plane multiplied by a weighting factor is defined as the cost function

$$
J=\left|\sum_{i=1}^{L} p\left(x_{i}, y_{i}, z_{0}\right) W\left(x_{i}, y_{i}, z_{0}\right)\right|^{2},
$$

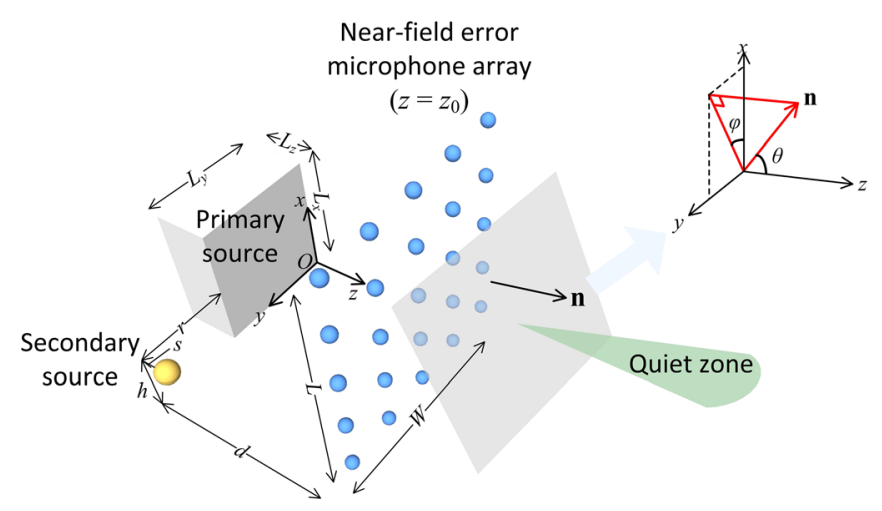

FIG. 1. (Color online) Schematic diagram of the near-field error microphone array. 
where the weighting factor

$$
W\left(x_{i}, y_{i}, z_{0}\right)=\mathrm{e}^{j\left(k_{x} x_{i}+k_{y} y_{i}+k_{z} z_{0}\right)}
$$

represents the phase shift from the point $\left(x_{i}, y_{i}, z_{0}\right)$ on the error microphone plane to the plane perpendicular to $\mathbf{n}$. If there are sufficiently many points on the plane, $P\left(k_{x}, k_{y}, z_{0}\right)$ will be minimized if $J$ is minimized. In the experiments, $\mathbf{n}$ is directed perpendicular to the microphone array and each weighting factor $W$ is unity. The cost function Eq. (4) can be simplified as the squared amplitude of the sum of the sound pressures at all the microphones.

In Eq. (4), $p\left(x_{i}, y_{i}, z_{0}\right)$ is the superposition of the primary and secondary sound pressure, which can be expressed in matrix form as

$$
\mathbf{p}=\mathbf{Z}_{\mathrm{pe}} q_{\mathrm{p}}+\mathbf{Z}_{\mathrm{se}} \mathbf{q}_{\mathrm{s}},
$$

where

$$
\mathbf{Z}_{\mathrm{pe}}=\left(\begin{array}{r}
Z_{\mathrm{p} 1} \\
Z_{\mathrm{p} 2} \\
\vdots \\
Z_{\mathrm{p} L}
\end{array}\right)
$$

is the acoustic transfer function vector from the primary source to the $L$ error points, and

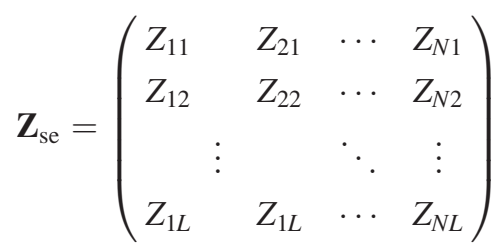

is the acoustic transfer matrix from $N$ secondary sources to the $L$ error points. Finally, $q_{\mathrm{p}}$ and $\mathbf{q}_{\mathrm{s}}$ are the strengths of the primary and secondary sources, respectively. The optimized strengths of the secondary sources can be obtained by minimizing Eq. (4) as

$$
\mathbf{q}_{s}=-\left(\mathbf{Z}_{\mathrm{se} 2}{ }^{\mathrm{H}} \mathbf{I} \mathbf{Z}_{\mathrm{se} 2}\right)^{-1} \mathbf{Z}_{\mathrm{se} 2}{ }^{\mathrm{H}} \mathbf{I} \mathbf{Z}_{\mathrm{pe} 2} q_{\mathrm{p}}
$$

where

$$
\mathbf{Z}_{\mathrm{pe} 2}=\left(\begin{array}{c}
Z_{\mathrm{p} 1} W\left(x_{1}, y_{1}, z_{0}\right) \\
Z_{\mathrm{p} 2} W\left(x_{2}, y_{2}, z_{0}\right) \\
\vdots \\
Z_{\mathrm{p} L} W\left(x_{L}, y_{L}, z_{0}\right)
\end{array}\right)
$$

and $\mathbf{Z}_{\mathrm{pe} 2}$ and $\mathbf{Z}_{\mathrm{se} 2}$ represent the acoustic transfer functions from the primary source and secondary sources to the projection of the error points on the plane perpendicular to $\mathbf{n}$. In Eq. (9), $\mathbf{I}$ is a $L \times L$ matrix where all the elements are 1 .

According to the spatial Fourier transform, $P\left(k_{x}, k_{y}\right.$, $z_{0}$ ) represents the amplitude of the sound propagation through the $z=z_{0}$ plane along the $\left(k_{\mathrm{x}}, k_{\mathrm{y}}, k_{\mathrm{z}}\right)$ direction, so the noise reduction can be guaranteed in this direction from the near to the far field if Eq. (4) is minimized, which is different from traditional local control. The idea used here is similar to that in planar near-field acoustical holography, where the wave field is measured on a planar surface to reconstruct the three-dimensional field. ${ }^{17}$ To the best of the authors' knowledge, this is the first time the spatial Fourier transform is applied in ANC for directivity control of sound radiation. For effective control performance, the suitable geometric configurations of the size of the microphone array, the microphone spacing and the distance between the array and the primary source are investigated in this paper. This sensing strategy improves the compactness of the ANC system and reduces the complexity and cost of the system. Because all the error signals are summed before feeding into an active controller, it reduces the requirements of the active controller from multiple error channels to a single error channel, and increases the convergence speed of the adaptive system.

\section{NUMERICAL SIMULATIONS}

As shown in Fig. 1, the size of the primary source in the simulations is $L_{x} \times L_{y} \times L_{z} \mathrm{~m}$, which is simulated by 216 monopoles evenly distributed within this volume, emitting sound energy at the same frequency but with random amplitudes and phases. The primary source is located within the cuboid from $(0,0,0)$ to $\left(L_{x}, L_{y},-L_{z}\right)$. A single secondary source is at $\left(h, L_{y}+r, s\right)$. The near-field error microphone array is on the $z=z_{0}$ plane, which is parallel to the primary source. The size of the microphone array is $L \times W \mathrm{~m}$. The distance between the array and the primary source is $d$, which is equal to $z_{0}$. The vector $\mathbf{n}$ represents the direction where noise reduction is required. The noise reductions at 7676 points evenly distributed on the $x=L_{x} / 2$ plane where $-7 \mathrm{~m} \leq y \leq 8 \mathrm{~m},-5 \mathrm{~m} \leq z \leq 15 \mathrm{~m}$ with an interval of $0.2 \mathrm{~m}$ are used to evaluate the performance of the system. 


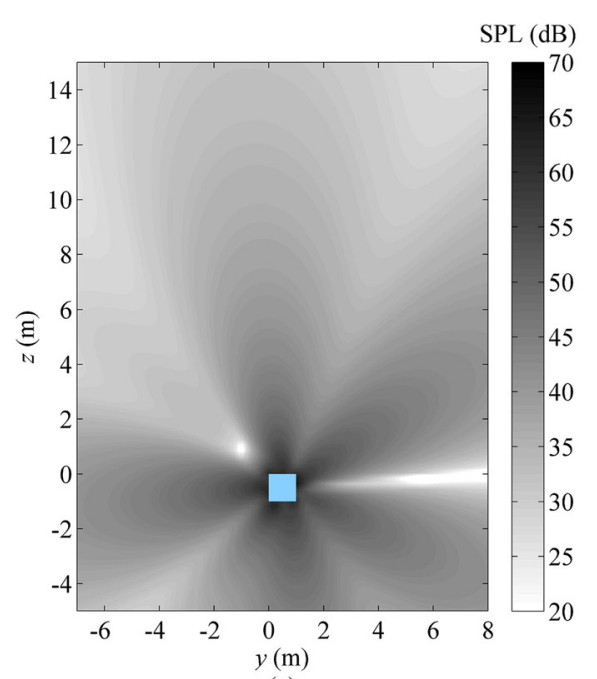

(a)

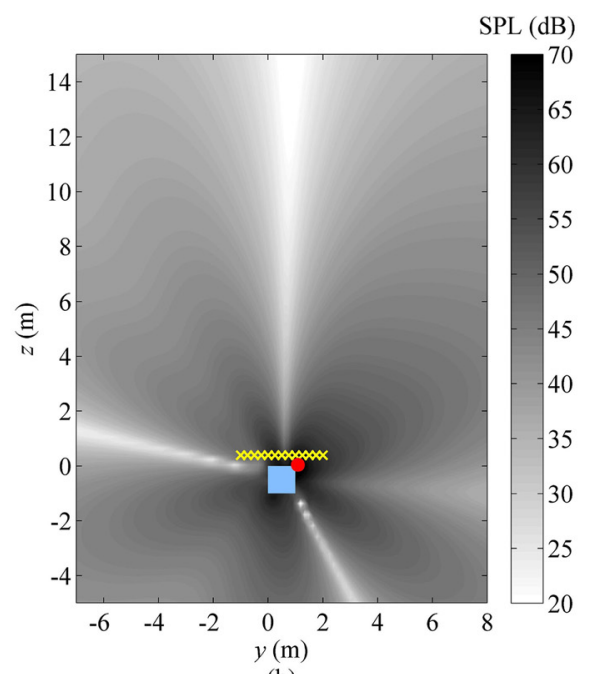

(b)

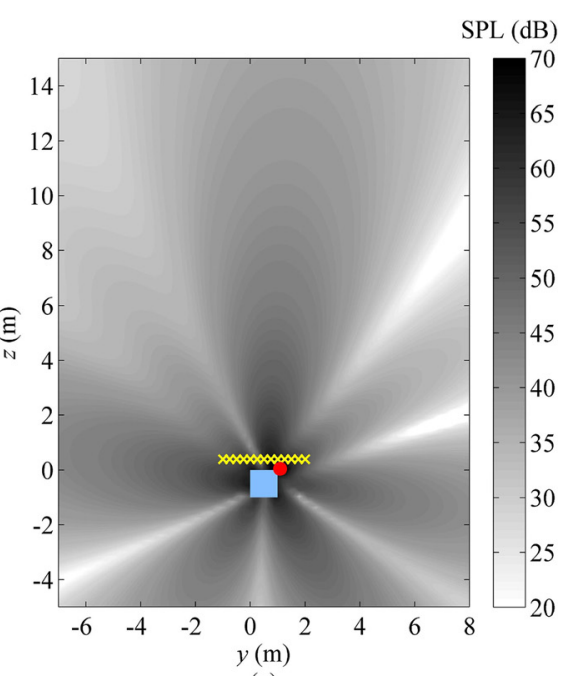

(c)

FIG. 2. (Color online) The SPLs in the evaluation plane at $340 \mathrm{~Hz}$ when $L_{x}=L_{y}=L_{z}=1 \mathrm{~m}, L=W=3 \mathrm{~m}$, and $d=0.4 \mathrm{~m}$, (a) the primary SPL, (b) the SPL with control when $\theta=0^{\circ}$ and $\varphi=90^{\circ}$, (c) the SPL with control when $\theta=30^{\circ}$ and $\varphi=90^{\circ}$. The blue rectangle represents the primary sources, the red circle represents the secondary source, and the yellow crosses represent error microphones.

\section{A. Directivity control}

The frequency of interest is $340 \mathrm{~Hz}$ and the wavelength $\lambda$ is $1 \mathrm{~m}$. The size of the primary source is set as $L_{x}=L_{y}=L_{z}=1 \mathrm{~m}$. The secondary source is $\lambda / 10$ away from the primary source $(r=0.0886 \mathrm{~m}, s=0.0500 \mathrm{~m})$. The primary sound pressure level (SPL) distribution in the evaluation plane is shown in Fig. 2(a). An array of $13 \times 13$ error microphones are evenly distributed on the plane of $L=W=3 \mathrm{~m}$ at $d=0.4 \mathrm{~m}$ in front of the primary source, and Eq. (9) is used to optimize $\boldsymbol{q}_{\mathrm{s}}$.

The distributions of the SPL with control are shown in Figs. 2(b) and 2(c). In Fig. 2(b), $\theta=0^{\circ}, \varphi=90^{\circ}$, and a quiet zone with more than $10 \mathrm{~dB}$ noise reduction is created directly in front of the error microphone array from the near to the far field. For $\theta=30^{\circ}$ and $\varphi=90^{\circ}$, the SPL with control is shown in Fig. 2(c). It is obvious that the error microphone array successfully creates a quiet zone in the target direction as well, which demonstrates the feasibility of the proposed error sensing strategy to achieve directivity control.

\section{B. Effects of the error microphone spacing}

Figure 3 shows the noise reduction (NR) when there are $4 \times 4,7 \times 7$, and $10 \times 10$ error microphones on a $3 \mathrm{~m} \times 3 \mathrm{~m}$ plane which is located $0.4 \mathrm{~m}$ in front of the primary source. The desired noise reduction direction is perpendicular to the error microphone plane, so $\theta=0^{\circ}, \varphi=90^{\circ}$. It can be seen that the system does not function until the distance between two neighboring microphones is reduced to $0.5 \mathrm{~m}$, which corresponds to $7 \times 7$ error microphones, as shown in Fig. 3(b). This indicates that there is a constraint on the microphone spacing.

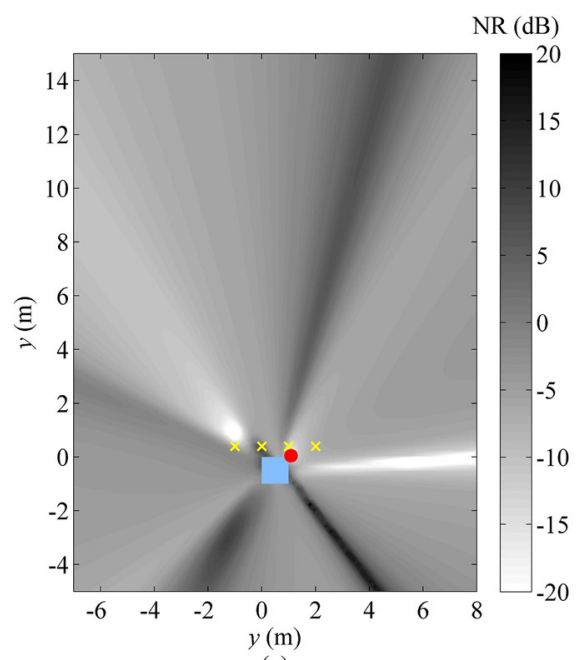

(a)

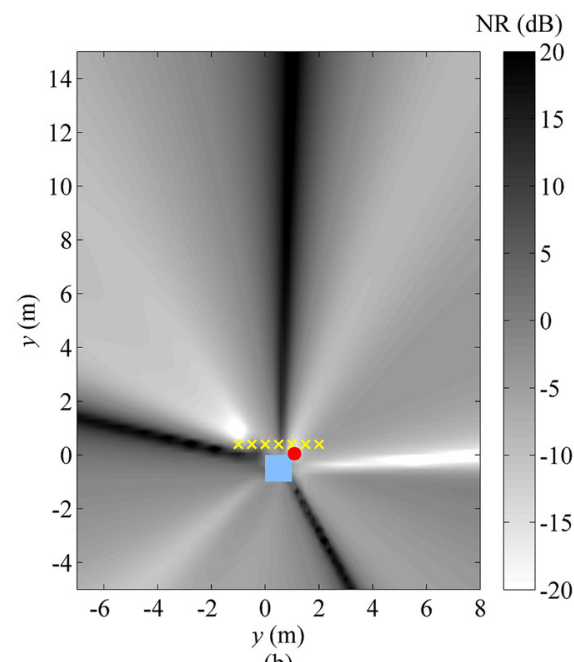

(b)

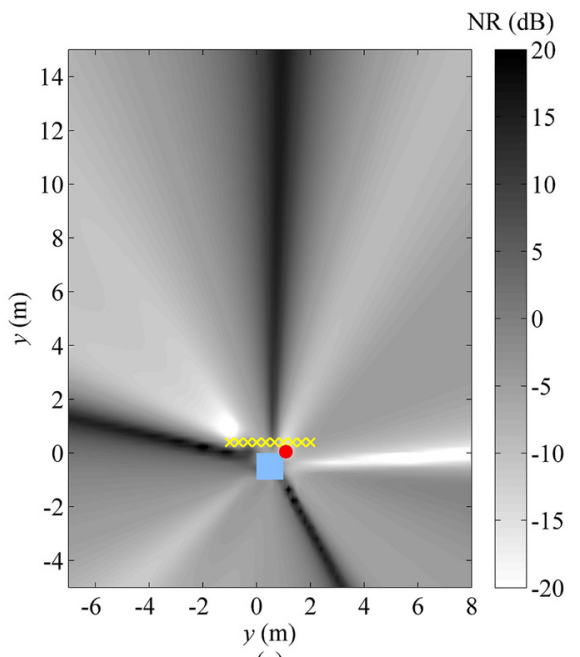

(c)

FIG. 3. (Color online) The distributions of NR in the evaluation plane at $340 \mathrm{~Hz}$ when $L_{x}=L_{y}=L_{z}=1 \mathrm{~m}, L=W=3 \mathrm{~m}, d=0.4 \mathrm{~m}, \theta=0{ }^{\circ}$, and $\varphi=90^{\circ}$ with: (a) $4 \times 4$ error microphones, (b) $7 \times 7$ error microphones, and (c) $10 \times 10$ error microphones. 

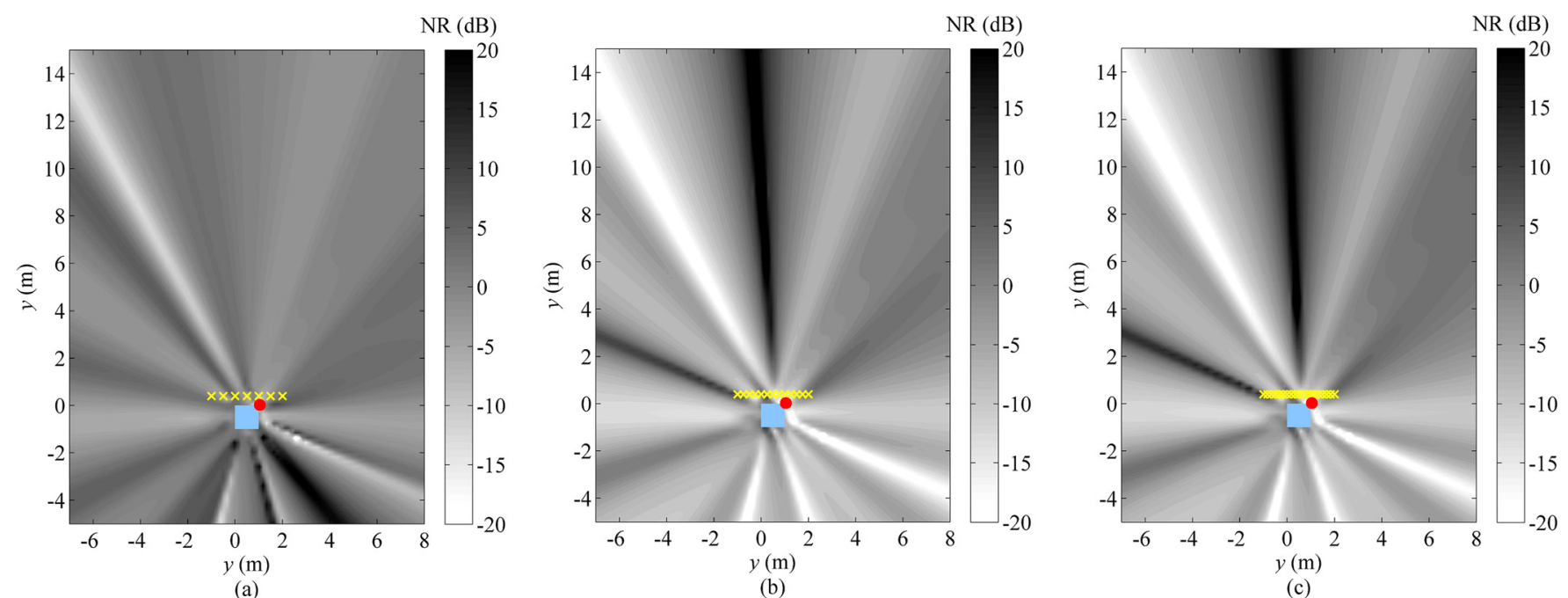

(b)

(c)

FIG. 4. (Color online) The distributions of NR in the evaluation plane at $680 \mathrm{~Hz}$ when $L_{x}=L_{y}=L_{z}=1 \mathrm{~m}, L=W=3 \mathrm{~m}, d=0.4 \mathrm{~m}, \theta=0^{\circ}$ and $\varphi=90^{\circ}$ with: (a) $7 \times 7$ error microphones, (b) $13 \times 13$ error microphones, and (c) $19 \times 19$ error microphones.

The NRs when there are $7 \times 7,13 \times 13$, and $19 \times 19$ error microphones on a $3 \mathrm{~m} \times 3 \mathrm{~m}$ plane at $680 \mathrm{~Hz}$ are shown in Fig. 4, in which the effective noise reduction area occurs when there are $13 \times 13$ error microphones or more. Table I lists the spacing requirements for effective active control with the error microphone array parallel to the primary source plane and $d=0.4 \mathrm{~m}$. The maximum allowed distance between neighboring microphones is $0.5 \mathrm{~m}$ for $340 \mathrm{~Hz}$ and $0.25 \mathrm{~m}$ for $680 \mathrm{~Hz}$, and it remains the same for different sizes of the microphone array, so it can be deduced that the maximum allowed microphone spacing is approximately half the wavelength of the frequency of interest in this case, which is similar to the rules in near-field acoustical holography (NAH) ${ }^{20}$

\section{Effects of the size of the near-field error microphone array}

Figure 5 shows the NR in the evaluation plane when the size of the primary source is $1 \mathrm{~m} \times 1 \mathrm{~m} \times 1 \mathrm{~m} . \theta$ and $\varphi$ are $0^{\circ}$ and $90^{\circ}$, respectively. The distance between neighboring error microphones is fixed at $\lambda / 4$, which satisfies the requirements mentioned above (less than $\lambda / 2$ ). The minimum size of the error microphone array is $2 \mathrm{~m}$, which is approximately twice the size of the primary source. This is consistent with the discussions in ISO 3744, where the measurement radius of the hemisphere is required to be equal to or greater than twice the characteristic source dimension for the determination of the sound power of the noise source. ${ }^{2}$

The NRs for $\theta=60^{\circ}$ and $\varphi=90^{\circ}$ with different array sizes are shown in Fig. 6. The size of the primary source is

TABLE I. The maximum allowed distance between neighboring microphones when $L_{x}=L_{y}=L_{z}=1 \mathrm{~m}$ and $d=0.4 \mathrm{~m}$.

\begin{tabular}{lccccc}
\hline \hline \multirow{2}{*}{$\begin{array}{l}\text { Maximum allowed } \\
\text { distance }(\mathrm{m})\end{array}$} & \multicolumn{4}{c}{ Size of the error microphone array $(L \times W)$} \\
\cline { 2 - 6 } & $2 \mathrm{~m} \times 2 \mathrm{~m}$ & $4 \mathrm{~m} \times 4 \mathrm{~m}$ & $8 \mathrm{~m} \times 8 \mathrm{~m}$ & $16 \mathrm{~m} \times 16 \mathrm{~m}$ \\
\hline Frequency (Hz) & 340 & 0.5 & 0.5 & 0.5 & 0.5 \\
& 680 & 0.25 & 0.25 & 0.25 & 0.25 \\
\hline \hline
\end{tabular}

$1 \mathrm{~m} \times 1 \mathrm{~m} \times 1 \mathrm{~m}$, and the distance between neighboring microphones is fixed as $\lambda / 6$, which is sufficient. It can be seen that the system starts functioning when $L=W=4 \mathrm{~m}$. Because $\theta=60^{\circ}$, the equivalent size of the array, which is the size of its projection on the plane perpendicular to $\mathbf{n}$ is $2 \mathrm{~m} \times 2 \mathrm{~m}$ and it is still twice the size of the primary source. It can be concluded from Figs. 5 and 6 that, to achieve effective directivity control, the minimum equivalent size of the error microphone array should be twice the size of the primary source.

\section{Effects of the distance between the microphone array and the primary source}

Figures 7(a) and 7(b) show the NRs at $340 \mathrm{~Hz}$ for a $7 \times 7$ error microphone array on a $3 \times 3 \mathrm{~m}$ plane (the microphone spacing is $\lambda / 2$ ) when $d=0.2$ and $0.4 \mathrm{~m}$. In the simulations, $L_{x}=L_{y}=L_{z}=1 \mathrm{~m}, \theta=0^{\circ}$, and $\varphi=90^{\circ}$. The NR in the desired direction is less than $10 \mathrm{~dB}$ when $d=0.2 \mathrm{~m}$ even though the error microphone array satisfies the requirements mentioned above; however, the NR increases when $d=0.4 \mathrm{~m}$, which means the distance between the microphone array and primary source affects the noise reduction performance as well.

The noise reduction when $d=0.2 \mathrm{~m}$ can be increased with more error microphones in the array. As can be seen from Fig. 7(c), when the microphone spacing is reduced to approximately $1 / 3$ the wavelength at $340 \mathrm{~Hz}$, the noise reduction increases. The closer the microphone array is to the primary source, the smaller the distance between neighboring microphones should be. This is similar to the rule in near-field acoustical holography, where the microphone spacing should be equal to or less than the distance between the sound source and microphone array because a large microphone spacing will result in insufficient spatial sampling and cause aliasing in reconstruction. ${ }^{20}$

To sum up, the simulations show that the proposed nearfield error sensing strategy can effectively create a quiet zone in the desired direction from the near to the far field. 


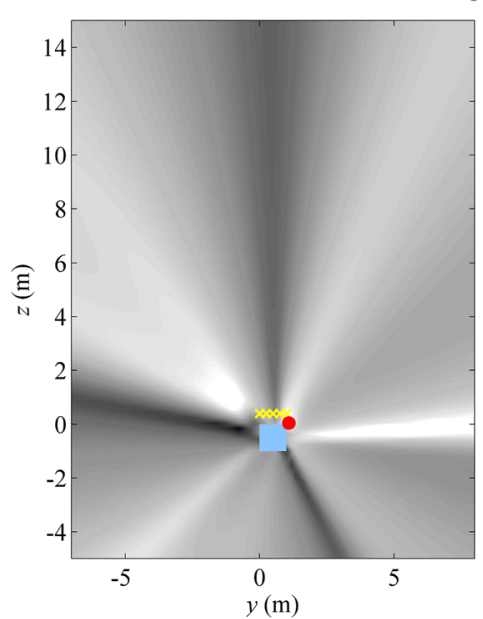

(a)

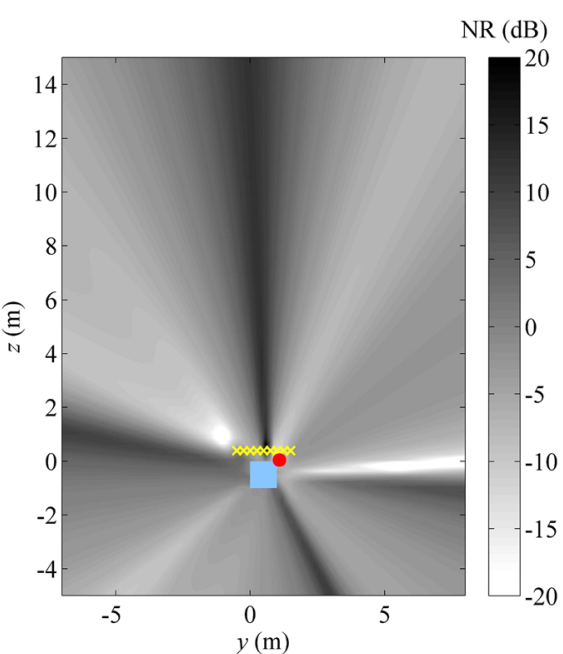

(b)

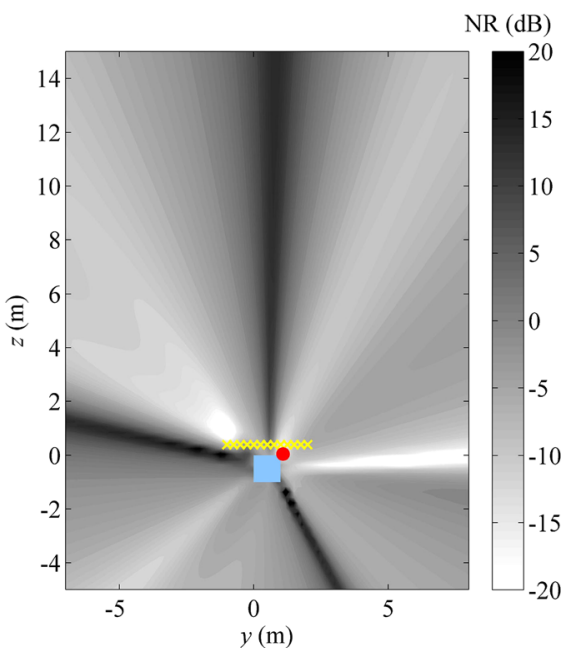

(c)

FIG. 5. (Color online) The distributions of NR in the evaluation plane at $340 \mathrm{~Hz}$ when the microphone spacing is $\lambda / 4, L_{x}=L_{y}=L_{z}=1 \mathrm{~m}, d=0.4 \mathrm{~m}, \theta=0^{\circ}$, and $\varphi=90^{\circ}$, (a) $L=W=1 \mathrm{~m}$, (b) $L=W=2 \mathrm{~m}$, (c) $L=W=3 \mathrm{~m}$.

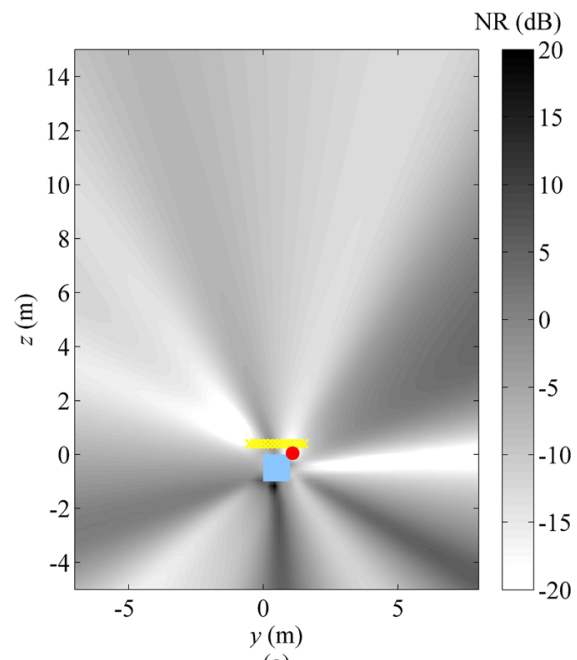

(a)

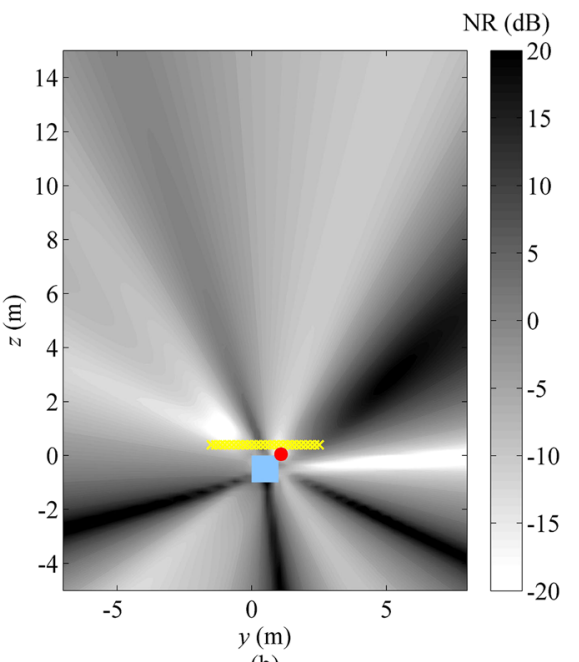

(b)

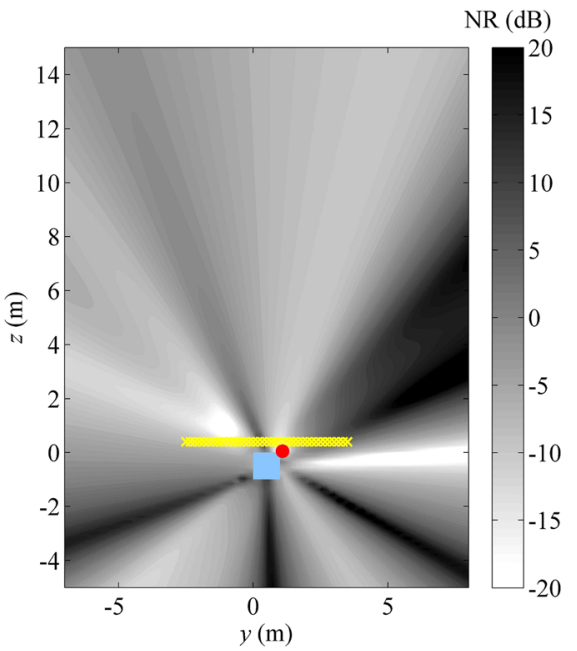

(c)

FIG. 6. (Color online) The distributions of $\mathrm{NR}$ in the evaluation plane at $340 \mathrm{~Hz}$ when the microphone spacing is $\lambda / 6, L_{x}=L_{y}=L_{z}=1 \mathrm{~m}, d=0.4 \mathrm{~m}, \theta=60^{\circ}$, and $\varphi=90^{\circ}$, (a) $L=W=2 \mathrm{~m}$, (b) $L=W=4 \mathrm{~m}$, (c) $L=W=6 \mathrm{~m}$.

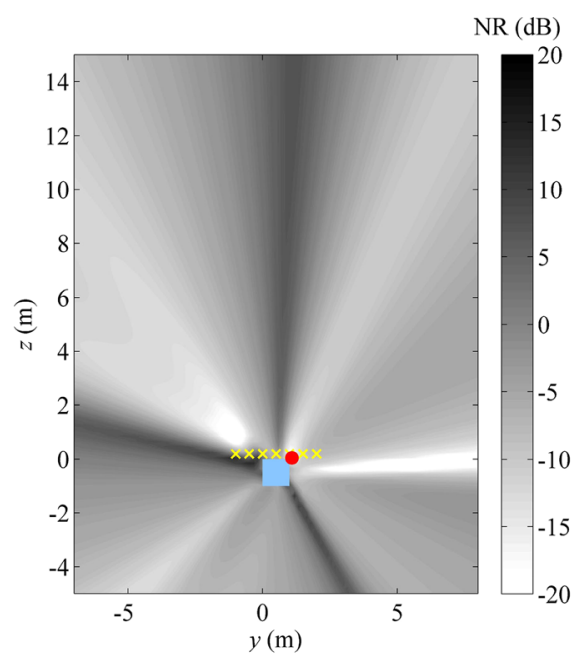

(a)

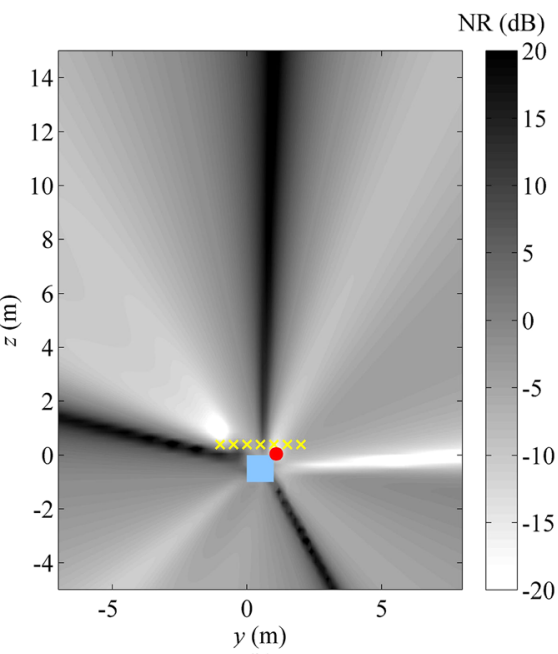

(b)

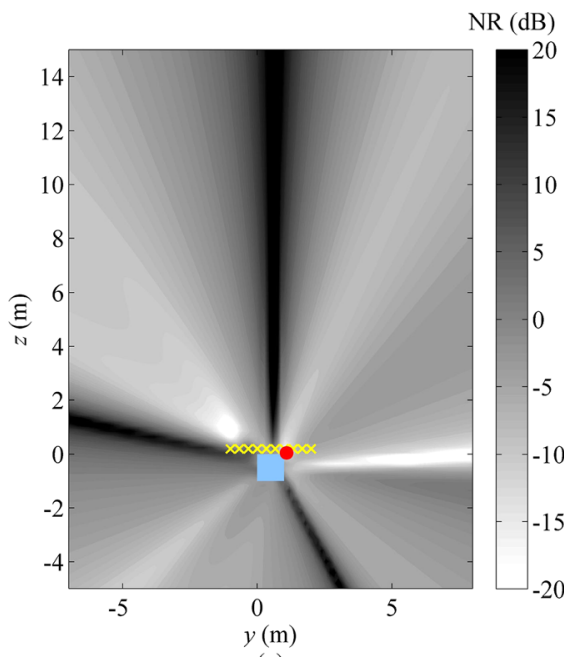

(c)

FIG. 7. (Color online) The distributions of NR in the evaluation plane at $340 \mathrm{~Hz}, L_{x}=L_{y}=L_{z}=1 \mathrm{~m}, L=W=3 \mathrm{~m}, \theta=0^{\circ}$, and $\varphi=90^{\circ}$, (a) $d=0.2 \mathrm{~m}$ and the microphone spacing is $\lambda / 2$, (b) $d=0.4 \mathrm{~m}$ and the microphone spacing is $\lambda / 2$, (c) $d=0.2 \mathrm{~m}$ and the microphone spacing is $\lambda / 3$. 


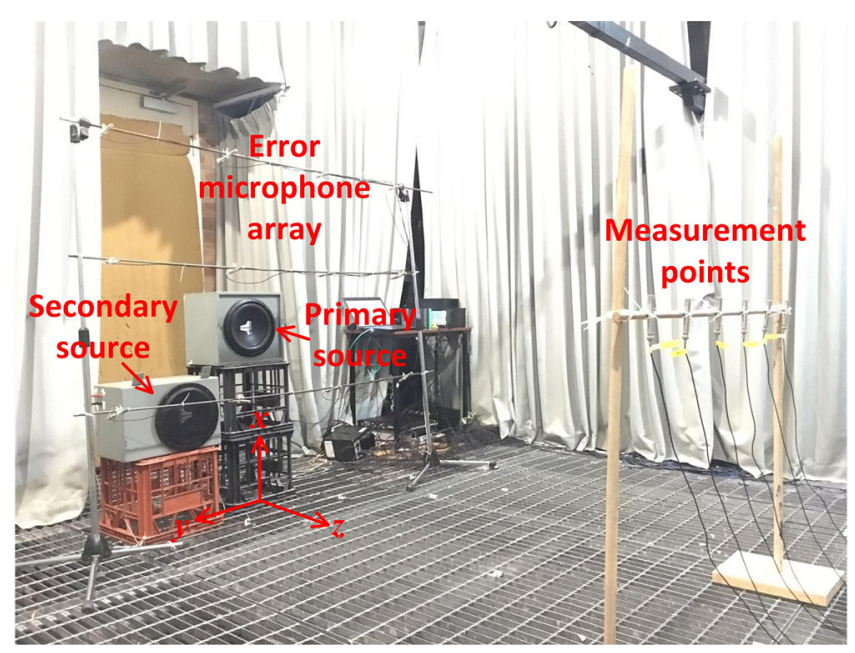

FIG. 8. (Color online) The experimental setup in an anechoic chamber.

The size of the array should be at least twice that of the primary source and the microphone spacing should be less than half the wavelength of the frequency of interest. A smaller microphone spacing is required when the array is close to the primary source.

\section{EXPERIMENTS}

The experimental setup in the anechoic chamber is shown in Fig. 8. Two loudspeakers are used to simulate the primary source and secondary source, respectively, and the distance between their centres is approximately $0.4 \mathrm{~m}$. The origin of the coordinate is at the floor. The centre of the primary source is at $(0,0) \mathrm{m}$ in the $y-z$ plane and it faces the positive $z$ direction. The near-field error microphone array consists of $4 \times 4$ microphones (microphone model: CHZ-213; preamplifier model: YG-201) evenly distributed on a $1.5 \mathrm{~m} \times 1.5 \mathrm{~m}$ plane, which is approximately $0.35 \mathrm{~m}$ in front of the primary source and parallel to the $x-y$ plane. All the microphones were calibrated using a B\&K 4231 Sound Calibrator and their phase differences are less than $2^{\circ}$ at $1000 \mathrm{~Hz}$. The frequency of interest is $240 \mathrm{~Hz}$. The signals picked up by the 16 error microphones are summed first and then fed into an active controller as a single input signal. A commercial adaptive ANC controller (TigerANC-II Lite) embedded with the FxLMS algorithm is used in the experiment. ${ }^{21,22}$

The SPL within a $2.8 \mathrm{~m} \times 2.2 \mathrm{~m}$ area in the $y-z$ plane $(-1.4 \mathrm{~m}<y<1.4 \mathrm{~m}, 0.6 \mathrm{~m}<z<2.8 \mathrm{~m})$ in front of the error microphone array at a height of $0.9 \mathrm{~m}$ with and without control and the corresponding noise reduction are shown in Fig. 9. The numerical simulation results obtained with the measured acoustic transfer functions and Eq. (9) are also included for comparison, which shows a local quiet zone within the direction perpendicular to the error microphone array. The experimental noise reduction in Fig. 9(c) is similar to the numerical simulation results in Fig. 9(f), which demonstrates the feasibility of the near-field error microphone array. There exist some differences between the simulation and experimental results because the system is hard to converge to the best condition in experiments due to the background noise.

Experiments with 16 error signals as 16 inputs to the multi-channel active controller were also carried out and the results are shown in Fig. 10. In this case, the cost function is the sum of the squared sound pressure at all the error microphones, which is the most commonly used cost function in active noise control. ${ }^{22}$ The experimental and numerical simulation results agree reasonably well in Fig. 10. It can be found by comparing Fig. 9(c) with Fig. 10(c) that the nearfield error microphone arrangement achieves much higher noise reduction in the desired direction than the traditional multi-channel active noise control. With all the error signals summed up, only a single-channel active controller is required, which minimizes the complexity and cost of the ANC system.

For broadband primary noise below $250 \mathrm{~Hz}$, Fig. 11(a) shows the error signal (the sum of the 16 signals picked up by the error microphones) with and without control, which shows apparent noise reductions within this frequency band. The noise reduction decreases above $200 \mathrm{~Hz}$ because of the limited taps of the control filters but the noise reduction is still more than $10 \mathrm{~dB}$. Figures $11(\mathrm{~b})-11(\mathrm{~d})$ show the SPLs at three different points in the far field, which are marked as asterisks in Fig. 12(a). It can be seen that the noise reduction in Fig. 11(c) is higher than that in Figs. 11(b) and 11(d)

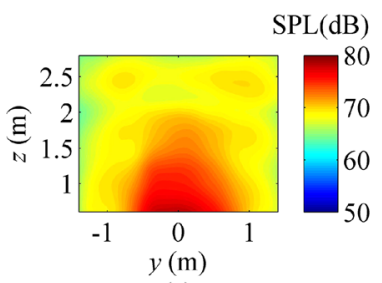

(a)

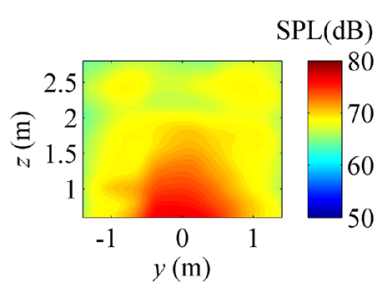

(d)

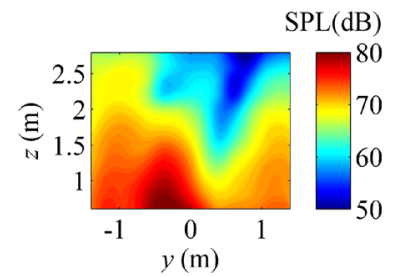

(b)

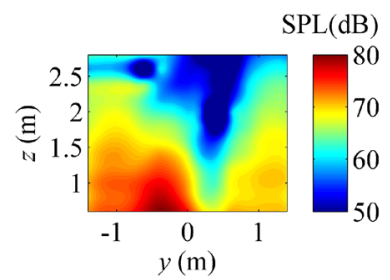

(e)

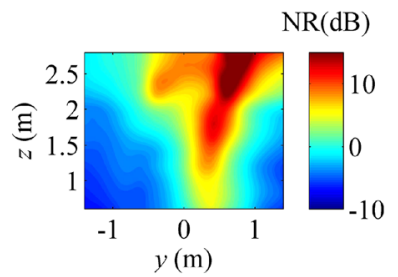

(c)

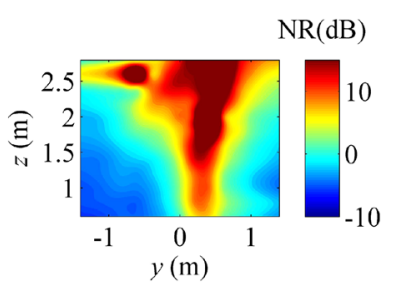

(f)
FIG. 9. (Color online) The SPLs and NRs in the $y-z$ plane achieved with the near-field error microphone array at $240 \mathrm{~Hz},-1.4 \mathrm{~m}<y<1.4 \mathrm{~m}, 0.6 \mathrm{~m}<z$ $<2.8 \mathrm{~m}$, experimental results: (a) the SPL without control, (b) the SPL with control, (c) the NR, and simulation results: (d) the SPL without control, (e) the SPL with control, (f) the NR. 


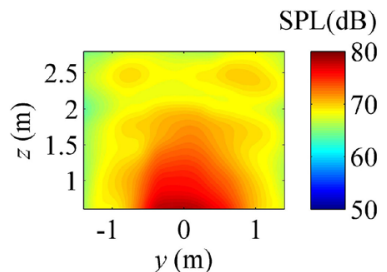

(a)

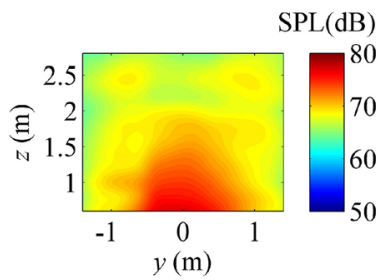

(d)

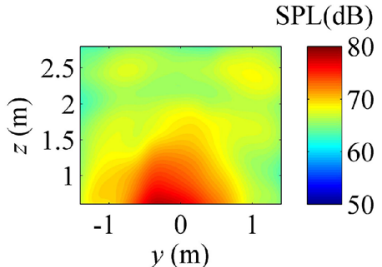

(b)

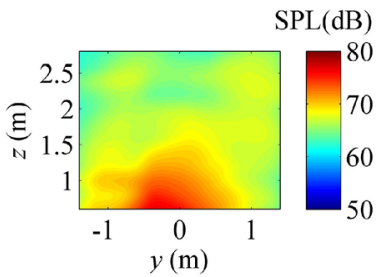

(e)

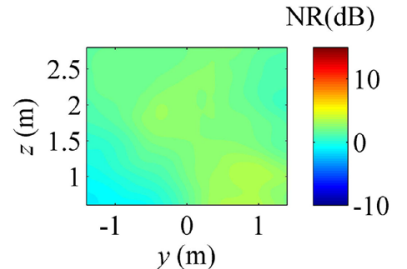

(c)

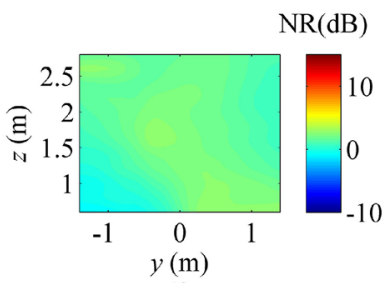

(f)
FIG. 10. (Color online) The SPLs and NRs in the $y-z$ plane achieved by minimizing the sum of the squares of the 16 error signals at $240 \mathrm{~Hz}$, $-1.4 \mathrm{~m}<y<1.4 \mathrm{~m}, 0.6 \mathrm{~m}<z<2.8 \mathrm{~m}$, experimental results: (a) the SPL without control, (b) the SPL with control, (c) the NR, and simulation results: (d) the SPL without control, (e) the SPL with control, (f) the NR. because the point investigated in Fig. 11(c) is in the direction perpendicular to the error microphone array. It demonstrates that directivity control of broadband noise can also be achieved with the error microphone array. The noise reduction in Fig. 11(c) is limited around $200 \mathrm{~Hz}$. This is partly due to the limitation of the physical configurations of the secondary source and error microphone array, as well as the frequency responses of the primary or secondary sources. Another reason might be the limited size of the anechoic chamber used for the experiment and that the boundaries are not $100 \%$ sound absorbent, which makes the experimental results a little different from the numerical simulation results in the free field. These reasons will be further investigated in the future.
Figures 12(a)-12(c) show the primary SPLs in the $1 / 3$ octave band with central frequencies 100, 125, and $160 \mathrm{~Hz}$, and Figs. 12(d)-12(f) show the corresponding noise reductions. It can be seen that the noise is significantly reduced in the direction perpendicular to the nearfield error microphone array in the $1 / 3$ octave band with the central frequencies 100 and $125 \mathrm{~Hz}$. Directivity control is also achieved for the central frequency $160 \mathrm{~Hz}$, but the noise reduction is smaller. More microphones in a larger plane might help improve the performance within this $1 / 3$ octave band.

For the error microphone array adjusted to have an angle of approximately $54^{\circ}$ between the $x-y$ plane, the SPLs and NRs in the $y-z$ plane $(-1.4 \mathrm{~m}<y<1.4 \mathrm{~m}, 1.0 \mathrm{~m}<z<2.8 \mathrm{~m})$

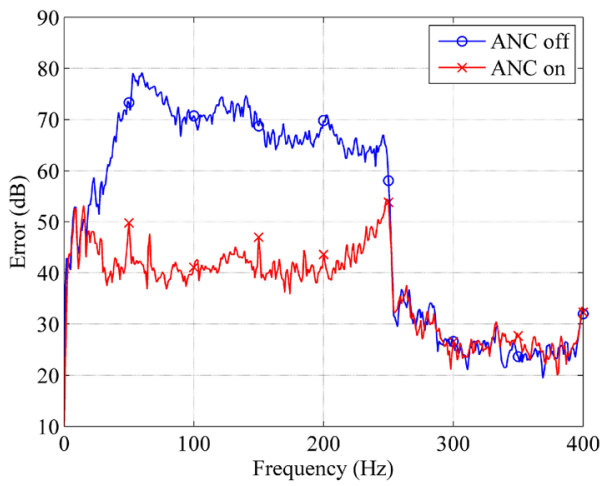

(a)

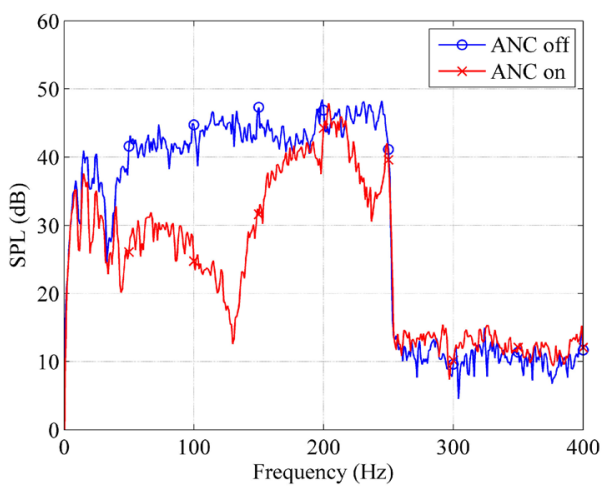

(c)

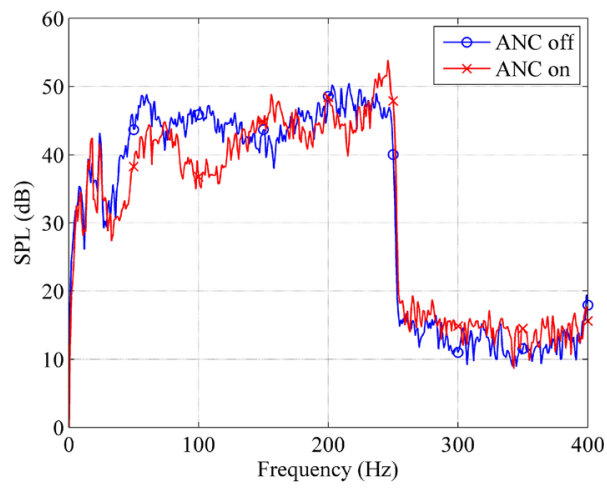

(b)

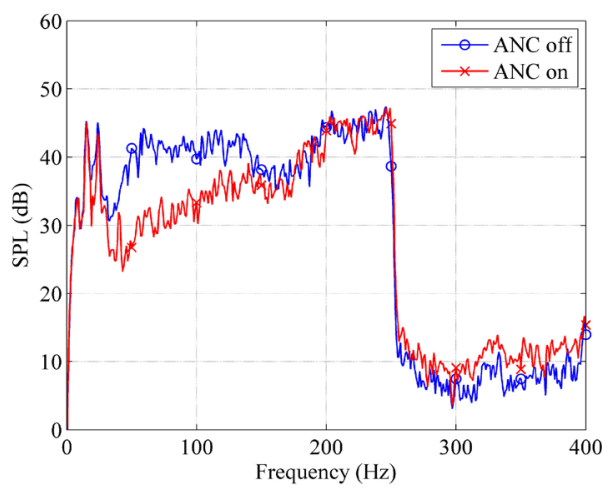

(d)
FIG. 11. (Color online) (a) The sums of 16 error signals with and without control, the SPLs at (b) $(0.9,1.2,1.7)$ $\mathrm{m}$, (c) $(0.9,0,2.6) \mathrm{m}$, and (d) $(0.9$, $-1.2,2.6) \mathrm{m}$ with and without control. 


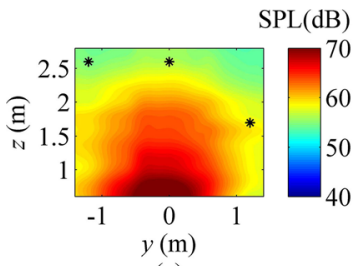

(a)

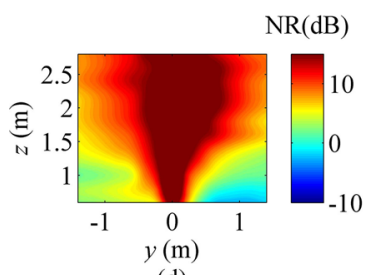

(d)

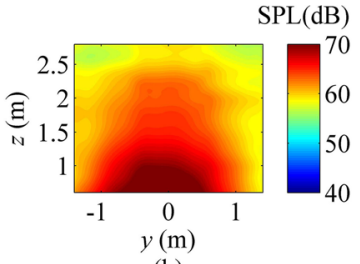

(b)

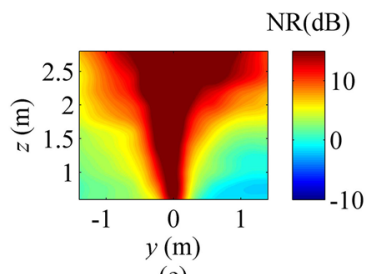

(e)

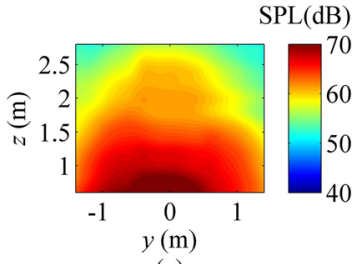

(c)

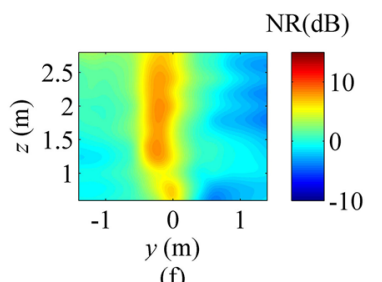

(f)
FIG. 12. (Color online) The primary SPLs in the $y-z$ plane in the $1 / 3$ octave bands with central frequencies, -1.4 $\mathrm{m}<y<1.4 \mathrm{~m}, \quad 0.6 \mathrm{~m}<z<2.8 \mathrm{~m}$ : (a) $100 \mathrm{~Hz}$, (b) $125 \mathrm{~Hz}$, (c) $160 \mathrm{~Hz}$; the NRs in the $1 / 3$ octave bands with central frequencies: (d) $100 \mathrm{~Hz}$, (e) $125 \mathrm{~Hz}$, (f) $160 \mathrm{~Hz}$. are shown in Fig. 13 with the noise reduction performance with 16 separate error signals included for comparison. Similar to Figs. 9 and 10, the near-field error microphone array achieves much better performance than 16 error signals. It can be seen from Fig. 13(c) that the effective noise reduction direction is changed and still remains approximately perpendicular to the error microphone array. Noise reduction in any direction can be achieved by placing the near-field error microphone array perpendicular to the direction or weighting the error signals according to Eq. (5) before summing them. In practical applications, the nearfield microphone array could be easily implemented by installing the array perpendicularly to the desired noise reduction direction. If that is inconvenient or there is not enough space for such a perpendicular installation, the error signals $e_{i}(t)$ can be convolved with the designed time delay filters first and then added up and fed into the active controller for minimization. The time delays are functions of $\theta, \varphi$, and $x$ and $y$ coordinates of the error microphones. Alternatively, the general complex weighting factors can be implemented in frequency domain. By minimizing the sum of the error signals multiplied by the weighting factors, the strengths of the secondary sources can be obtained and then transformed into time domain with inverse fast Fourier transform (IFFT) and fed into the secondary sources.

\section{CONCLUSIONS}

In order to reduce sound radiated to a certain direction, a new near-field error sensing strategy was developed by placing a planar error microphone array near the primary source and minimizing the weighted sum of the sound pressure at all these points. The weighting factor corresponds to the phase shift from the error microphones to the plane perpendicular to the direction where noise reduction is required. The effectiveness of the proposed strategy to achieve directivity control from the near to the far field was demonstrated with numerical simulations and the requirements on the near-field error microphone array were discussed. It was found that the microphone spacing should be no more than half the wavelength of the frequency of interest. The minimum equivalent size of the array is approximately twice the size of the primary source when the secondary source is close to the primary source. The distance between the error microphone array and primary source also affects the noise reduction performance and smaller microphone spacing is required when the array is close to the primary source. Experiment results in the anechoic chamber demonstrated the feasibility of the proposed method. The possibility of using microphone arrays of other shapes such as spherical microphone arrays to achieve directivity or even global control will be investigated in the future.

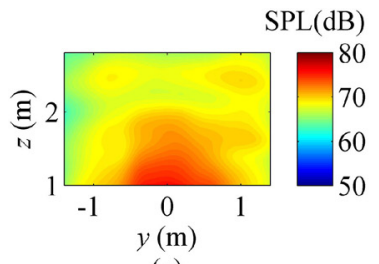

(a)

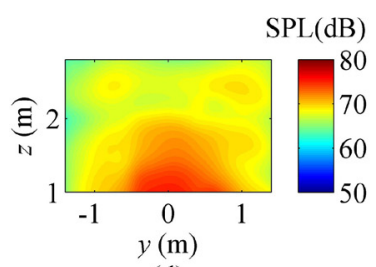

(d)

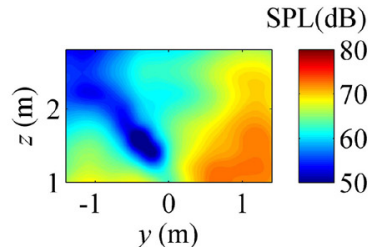

(b)

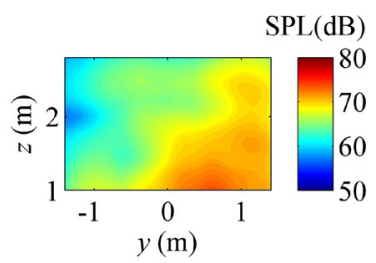

(e)

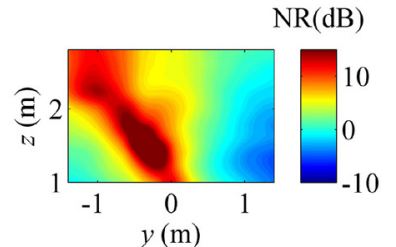

(c)

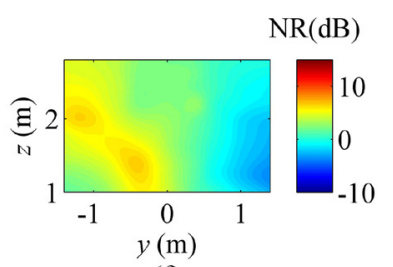

(f)
FIG. 13. (Color online) The SPLs with and without control and the NRs in the $y-z$ plane at $240 \mathrm{~Hz}$ when the angle between the near-field microphone plane and the $x-y$ plane is $54^{\circ},-1.4 \mathrm{~m}$ $<y<1.4 \mathrm{~m}, \quad 1.0 \mathrm{~m}<z<2.8 \mathrm{~m}$, the results for the error microphone array: (a) the SPL without control, (b) the SPL with control and (c) the NR; the results for 16 errors: (d) the SPL without control, (e) the SPL with control and (f) the NR. 


\section{ACKNOWLEDGMENTS}

This research was supported by the National Science Foundation of China (11474163) and under the Australian Research Council's Linkage Projects funding scheme (LP140100987).

${ }^{1}$ P. A. Nelson, A. R. D. Curtis, S. J. Elliott, and A. J. Bullmore, "The minimum power output of free field point sources and the active control of sound," J. Sound Vib. 116(3), 397-414 (1987).

${ }^{2}$ ISO 3744:2010, "Acoustics-Determination of sound power levels and sound energy levels of noise sources using sound pressure-Engineering methods for an essentially free field over a reflecting plane" (International Organization for Standardization, Geneva, Switzerland, 2010).

${ }^{3}$ A. Berry, X. Qiu, and C. Hansen, "Near-field sensing strategies for the active control of the sound radiated from a plate," J. Acoust. Soc. Am. 106(6), 3394-3406 (1999).

${ }^{4}$ F. Niu, H. Zou, X. Qiu, and M. Wu, "Error sensor location optimization for active soft edge noise barrier," J. Sound Vib. 299(1), 409-417 (2007).

${ }^{5}$ J. Julliard, H. Antoine, C. Lozachmeur, and A. Roure, "Active control of directivity of fan tones noise," in RTO AVT Symposium on Active Control Technology for Enhanced Performance Operational Capabilities of Military Aircraft, Land Vehicles and Sea Vehicles, Braunschweig, Germany (2000).

${ }^{6} \mathrm{~B}$. Rafaely, "Spherical loudspeaker array for local active control of sound," J. Acoust. Soc. Am. 125(5), 3006-3017 (2009).

${ }^{7}$ X. Qiu and S. Zhao, "Active control of the directivity of the sound diffraction from barriers," in Proceedings of the 22nd International Congress on Sound and Vibration, Florence (2015).

${ }^{8}$ Z. Lin, L. Zhang, X. Qiu, and J. Pan, "An integrated passive and active control system for reducing haul truck noise," in Proceedings of InterNoise, Melbourne (2014).

${ }^{9}$ C. Hansen, S. Snyder, X. Qiu, L. Brooks, and D. Moreau, Active Control of Noise and Vibration (CRC Press, Boca Raton, FL, 2012).
${ }^{10}$ B. M. Shafer, K. L. Gee, and S. D. Sommerfeldt, "Verification of a nearfield error sensor placement in active control of compact noise sources," J. Acoust. Soc. Am. 127, EL66-EL72 (2010).

${ }^{11}$ A. C. Zander and C. H. Hansen, "A comparison of error sensor strategies for the active control of duct noise," J. Acoust. Soc. Am. 94(2), 841-848 (1993).

${ }^{12}$ X. Qiu, C. H. Hansen, and X. Li, "A comparison of near-field acoustic error sensing strategies for the active control of harmonic free field sound radiation," J. Sound Vib. 215(1), 81-103 (1998).

${ }^{13}$ J. Garcia-Bonito, S. J. Elliott, and C. C. Boucher, "Generation of zones of quiet using a virtual microphone arrangement," J. Acoust. Soc. Am. 101(6), 3498-3516 (1997).

${ }^{14}$ A. P. Berkhoff, "Control strategies for active noise barriers using nearfield error sensing," J. Acoust. Soc. Am. 118(3), 1469-1479 (2005).

${ }^{15}$ C. R. Fuller, C. Papenfuss, and T. D. Saux, "Active-passive control of portable generator set radiated noise," in Proceedings of Acoustics 2012, Fremantle, Australia (2012).

${ }^{16}$ D. Peterson, A. C. Zander, B. S. Cazzolato, and C. H. Hansen, "Optimal virtual sensing for active noise control in a rigid-walled acoustic duct," J. Acoust. Soc. Am. 118(5), 3086-3093 (2005).

${ }^{17}$ C. D. Peterson, A. C. Zander, B. S. Cazzolato, and C. H. Hansen, "A moving zone of quiet for narrowband noise in a one-dimensional duct using virtual sensing," J. Acoust. Soc. Am. 121(3), 1459-1470 (2007).

${ }^{18}$ C. D. Peterson, R. Fraanje, B. S. Cazzolato, A. C. Zander, and C. H. Hansen, "A Kalman filter approach to virtual sensing for active noise control," Mech. Syst. Signal Process. 22(2), 490-508 (2008).

${ }^{19}$ E. G. Williams, Fourier Acoustics: Sound Radiation and Nearfield Acoustical Holography (Academic, New York, 1999).

${ }^{20} \mathrm{~S}$. F. Wu, "Methods for reconstructing acoustic quantities based on acoustic pressure measurements," J. Acoust. Soc. Am. 124(5), 2680-2697 (2008).

${ }^{21}$ Information on ANC controller available at http://www.antysound.com/ Controller.html (Last accessed 3 May 2018).

${ }^{22} \mathrm{P}$. Nelson and S. Elliott, Active Control of Sound (Academic, New York, 1992). 